\title{
Engaging young people with STEAM: Destinazione Luna
}

\author{
Sara Ricciardi \\ INAF Italian National Institute for Astrophysics \\ OAS Bologna, Via Gobetti 101 \\ email: sara.ricciardi@inaf.it
}

\begin{abstract}
On the occasion of celebration of moon landing (2019) we designed a set of educational activities for the youngest, based on the moon. We wanted to talk, play and engage young people reflecting and enjoying different points of view and demystifying the idea of science and scientists in a personal and meaningful journey. After this year of experimentation we also engaged with public schools co-designing and tailoring those activities despite the current sanitarian crisis. We will describe a physical artifact called 'Lunatario' and its $3 \mathrm{~d}$ printable version together with cross-disciplinary educational activities and our tentative documentation. We will also describe how, with the help of a very diverse team, we embedded other media in our moon exploration working in particular with picture books and animation. We believe this is a great way to deeply engage young people with STEAM in a democratic way.
\end{abstract}

Keywords. STEM education, constructionism, Moon

\section{Introduction}

We believe INAF as a Public Institute could play a main role in developing digital and technological skills in education, as required by the Digital Education Action Plan [European Commission (2018)] of the European Commission also in a lifelong-learning perspective for teachers and educators and in general to cope with the needs of the so called "knowledge-based society", [Lisbon's strategy (2000)]. In the year of the anniversary of the Man on the Moon we feel the "moral obligation" to develop educational practices and outreach activities linked with such an amazing event in the history of humankind. Because of the universality of this event and the personal feeling often connected to the historical moment and to Space Exploration in general we had a great opportunity to engage with people that are not necessarily in our typical audience. We embraced this challenge and we chose to unfold this story in multiple ways, defining strategies and contents depending on the different audiences and occasions. To manage this challenge we partnered with the Cineteca di Bologna,a cultural institution and a world-renowned mecca for cinephiles, with Hamelin, a cultural association working with children and young adults through literature, comics, illustration and cinema, with Istituto Comprensivo 12, a public school complex (kindergarten to middle school) codesigning and testing the educational activities. We hosted a variety of activities: from very cozy workshops to giant events such as the final open air movie night with more than 5000 people in the main square of Bologna. For the rest of the paper we will focus on Destinazione Luna kids a portfolio of educational activities for kinder garden and primary schools.

(C) The Author(s), 2021. Published by Cambridge University Press on behalf of International Astronomical Union. This is an Open Access article, distributed under the terms of the Creative Commons Attribution licence (http://creativecommons.org/licenses/by/4.0/), which permits unrestricted re-use, distribution, and 

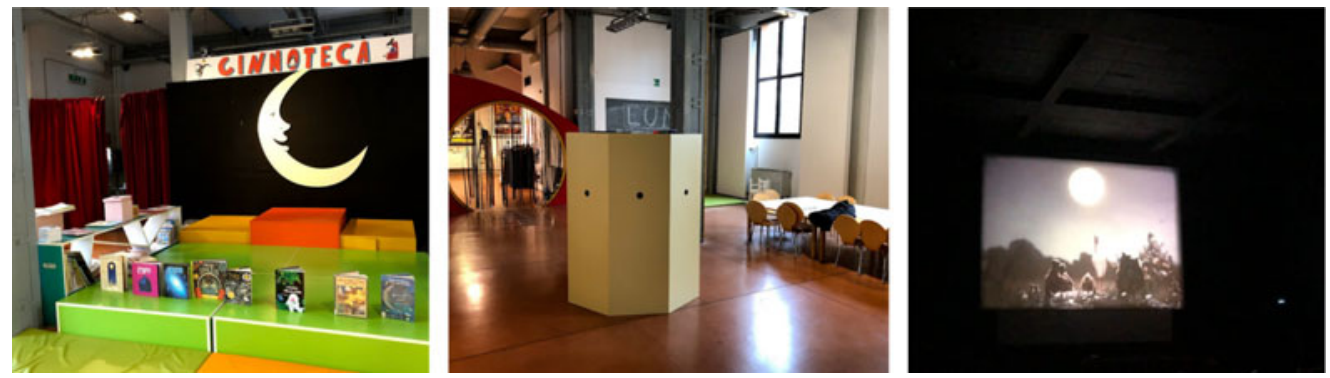

Figure 1. The dedicated spaces for the 3 phases of Destinazione Luna KIDS as implemented at Cineteca di Bologna: the collective reading of picture books, the wooden Lunatario and the animation in Sala Cervi.

\section{Destinazione Luna Kids (DLK)}

Thanks to DLK, between January and April 2019, we managed to reach around 12 schools in the Bologna area and to meet over 300 girls and boys aged between 3 and 6 . The twofold objective of our didactic experiment is to follow the children in a guided observation of the moon-object focusing on our perception and ability to infer the shape of the 3D object lighted by a beam of light and to stimulate their curiosity towards astrophysics; on the other hand we look at the moon through the lens of imagination, viewing - in the iconic Sala Cervi - short films dedicated to the Moon. Given the available time frame, the logistics and taking into account the cognitive development of the children at this stage we structured the workshop in a single two hours appointment and we provided follow-up materials for the teachers. We unrolled the party in three phases: a first moment dedicated to the collective reading of picture books, a second part devoted to the observation of the Moon and its phases with the wooden Lunatario and finally the experience of the movie theater (Fig. 1). We also developed other educational activities for the deployment of DLK in schools including a portable lunatario. All the resources are available on the INAF website devoted to innovation in education (PLAY website https://play.inaf.it/destinazione-luna/ multiple languages)

\subsection{From STEM to STEAM to STREAM to STE*M: picture books and movie}

The classical definition of STEM (Science Technology Engineering and Math) used to group together those considered hard-science has been often replaced by the acronym STEAM. The A is for Art and basically represents the need to use STEAM disciplines as a true form of self expression and so self determination in your own learning because historically Art is connected with this idea. Also STEM disciplines are a way to selfexpress; even though scientific method is quite rigidly coded and the accuracy of its application grants the credibility itself of our research, there is an enormous space for creativity even if often this is not visible as much as in the artists' work. Another reason why not to segregate STEM disciplines from Art is the audience. Children are still not so affected by the useless atomization of knowledge in disciplines and they often learn in a "Leonardesque" fashion without worrying too much about disciplines. Finally also the research communities are recognizing disciplines as aggregate around questions, such as the twentieth-century sciences (cosmology, ecology, neuroscience) instead of sciences historically organized around funds, editors and University departments [Morin 1999]. Recently with the proposal of STREAM we add one more layer: the $\mathrm{R}$ is for reading and wRiting. Advocates of STREAM see literacy as an essential part of a wellrounded curriculum, as it requires critical thinking as well as creativity. This may be 
true but we connected STEAM and reading for other reasons; in our previous experiences we found that using a familiar object such as a picture book can help to spark the initial interest through a familiar and well known object and will help to keep a very short psychological distance between the children and the scientist or educator. The picture book is, in our opinion, the perfect object of transition from a familiar and protected world to the "realm of STEM" that for someone could be intimidating. What we want definitely to avoid is that the "WOW-effect" (intended as the feeling of wonder and excitement happening when people get to explore astrophysics) will become a sort of psychological obstacle; we believe that this "wonder" is something that is not always positive for everyone in the classroom not because those feelings are negative per se but because children could feel they are not smart enough to be involved in such wonderful (but maybe distant) ideas. Loads of evidences are collected showing e.g. the connection between self prejudice in STEM learning [Bian L., Leslie S.J., Cimpian A. 2017]. For all those reasons we believe the familiar picture book is one of the best trick to start a deep and passionate discussion about science that is not intimidating. We strongly believe in active, self-directed approaches in education and this has very often led us to value activities where the content cannot be 'transferred' or directly told or shown to the kids. Competences and beliefs cannot be taught but need a different medium (process) where they are achieved through the active construction of new and often unexpected meanings. This is always the case with modern picture books, where the meaning is not laid down in front of the reader but needs to be reconstructed in the composition of text and pictures, in the actual dynamics of the pages, where every new opening requests the active participation of the reader building a personal experience. Pictures do not only add to the text an iconography but are always necessary in the composition and are part of a whole. In the same way, in astrophysics, images are not only an explanatory addendum to the scientific content of research but are the place where the meaning is hidden and needs to be constructed through the test and the abstraction and the understanding of a scientific idea. Similar reflection guided the selection process of the short films taking care also principles of universal design e.g. we choose short film for its hermetical structure that makes it closer to poetry, we chose films without dialogues to facilitate kids' fruition. The full bibliography and filmography with guidelines are available online and on the booklet we publish.

\subsection{Lunatario design}

Astrophysics is largely a history of light even if recently gravitational waves are enriching our "view". Moreover light shapes our perception. We see things and we build models of the world around us observing the light scattering on objects. If we can't touch those objects, e.g. looking to an astronomical object as the Moon, this scattering is the only clue we have to construct our knowledge. So we decided to design an artifact that will explore this idea allowing us to visually explore the Moon and mimicking the actual observation from Planet Earth. As astrophysicists we always build artifacts [Papert 1980] such as telescopes or satellites to extract and share knowledge. Our attempt was to design an object that will allow us to tinker with the ideas of light, perception and our place in the (close) Universe. This artifact is basically a magic box, a platform for visual experiments: an octagonal wooden box (1.2 $\mathrm{m}$ of diameter) with 8 peeping holes to visually explore a $3 \mathrm{D}$ printed white moon fixed on the ceiling with a fishing string so in the dark it would appear suspended. With a light source (a torch positioned in one of the holes) the kids were able to experiment and "almost touch" moon phases. The facilitation of the Lunatario experience was playful,lightly guided and the kids build a common knowledge sharing insights between peers. The children were invited to explore the Lunatario; 

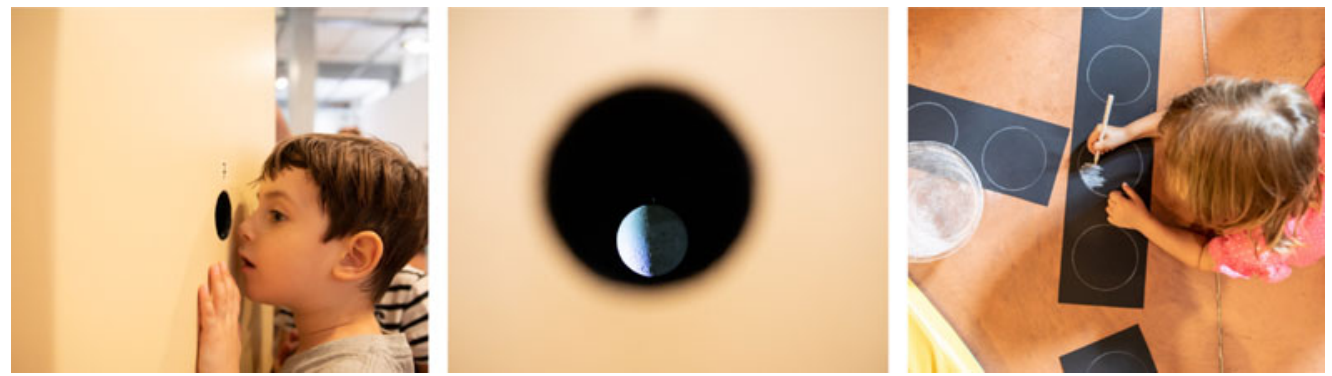

Figure 2. A children looking at the Lunatario and his view. A little girl materializes her perceptions and ideas with paper and pencils.

freely playing and reflecting together they had the chance to independently guess what was inside the box, and how the appearance of the 3D printed moon changed according to the relative position between the light source and their position. When question or perplexity rose the educator encouraged the children to express their own idea, reformulating if needed and easing the share of the insights. When a first consensus was reached the educator changed the position of the Sun (torch). This gesture opens up more questions and thoughts and another phase of discussion. With enough time for exploration children build the idea of the importance of the relative position between the sun (torch) and the observer. We found very useful to provide some craft materials to "materialize concept" and thing a bit away from the box. For this purpose we provided black cards and white pencils so the children could choose to reproduce one or more "moon phases". This reflexive gesture was integrated as needed with new observations or "life drawing" with an eye on the Lunatario and an eye on the drawing. With the youngest this was very important because the concept of right/left and up/down dark/light may be not yet consolidated (Fig. 2).

We observed that this small and familiar moon sparkles new curiosity in children that immediately recall episodes of moon's observation and they express the desire to observe the real Moon again. The Lunatario is hopelessly exposed to the curiosity of the observers thanks to those small holes, the only way to reveal the unknown content. It's interesting to underline the analogy with so many optic toys and pre-cinematographic apparatus: from the chamber obscura to the zootrope, from the stereoscope to the Edison's kinetoscope. After all, the wonder, the "magic of vision", are the key elements in the cinema itself with reference to the relationship with the viewer. We also try other experimentation using Lunatario as a first playful step for a more complex workshop. As an example during the "Festival del Cinema ritrovato" Bologna June 2019 we animated the moon seen in the Lunatario using a stop motion app (Stopmotion Studio App www.cateater.com). The design of this exhibit and the associated activities are still evolving and they will be proposed and tested in other environments and targeting different audiences. We also prototyped a version of this artifact with Digital Fabrication (3D printing) for portability, to allow different solid figures in the Lunatario and with the hope to illustrate the pedagogical possibility 3D printing open up and how this technology could serve to construct knowledge and own it.

\section{References}

European Digital Education Action Plan, COM/2018/022 final 2018

European Council Lisbon's strategy 2000.

Morin E. 1999 Seuil La Tête bien faite. Repenser la réforme, réformer la pensée

Bian L., Leslie S.J., Cimpian A. 2017 Science Vol. 355, Issue 6323, pp. 389-391

Papert S.1980 Basic Books Mindstorms: Children, Computers, and Powerful Ideas 